\title{
A celiac case mimicking mitochondrial neurogastrointestinal encephalomyopathy (MNGIE)
}

\author{
Erhan Aksoy ${ }^{1}$, Serap Tiraş-Teber², Gülhis Deda² \\ ${ }^{1}$ Department of Pediatric Neurology, Dr. Sami Ulus Maternity and Childrens' Health and Diseases Training and Research \\ Hospital, ${ }^{2}$ Division of Pediatric Neurology, Department of Pediatrics, Ankara University Faculty of Medicine, Ankara, Turkey. \\ E-mail: aksoyerhan24@gmail.com
}

Received: 12th May 2016, Revised: 24th May 2016, Accepted: 18th July 2016

\begin{abstract}
SUMMARY: Aksoy E, Tıraş-Teber S, Deda G. A celiac case mimicking mitochondrial neurogastrointestinal encephalomyopathy (MNGIE). Turk J Pediatr 2017; 59: 662-665.

Celiac disease (CD) is a chronic disease involving a number of systems in addition to gastrointestinal tract. Although not clear, it has been supposed that the neurological symptoms of CD develop due to immune-mediated mechanisms. In this paper, we present a rare case diagnosed with CD at 12 years of age, and presented with a clinical picture resembling mitochondrial neurogastrointestinal encephalomyopathy (MNGIE). She had onset of her neurological symptoms at the age of 6 years, they progressed despite various therapies, and she became wheelchair-bound.
\end{abstract}

Key words: celiac disease, MNGIE, neurological involvement.

Celiac disease (CD) is a chronic disorder triggered by gluten components such as wheat, barley and rye. HLA-DQ2/DQ8 genetic sensitivity is important in pathogenesis. The incidence of $\mathrm{CD}$ is $1 \%^{1,2}$.

Although the target organ is intestine, extraintestinal organ involvement is seen in $20-30 \%$ of the patients. Thyroid, skin, pancreas, hearth, muscles, bones, reproductive system, liver, and peripheral and central nervous systems may be involved $^{2-4}$. The gastrointestinal tract symptoms are predominant in infants and children; but extra-intestinal involvement is seen at older ages. Therefore, CD is not included in the differential diagnosis of the older patients with extra-intestinal involvement, and the diagnosis could easily be missed ${ }^{3-5}$.

In this paper, we present a rare case presented with the symptoms of mitochondrial neurogastrointestinal encephalomyopathy (MNGIE), diagnosed with CD, and continued to have symptoms although on gluten-free diet.

\section{Case Report}

A 12-year-old girl was consulted to our department with the complaint of ptosis, and difficulty in talking. The patient was wheelchairbound. She had nausea and vomiting in the previous 2 months.

The complaints of the patient started at the age of 6 years with left sided dystonic movements. She was wheelchair-bound at 8 years of age, and had difficulty in talking, and ptosis. She had a brain magnetic resonance imaging (MRI) at that time, and hyperintense signals were seen in bilateral putamens and caudate nuclei. MR spectroscopy revealed lactate peaks, she was diagnosed with mitochondrial cytopathy at 9 years of age, and put on coenyzme Q10, lipoic acid, and L-Dopa (Fig. 1, 2). At that time, the blood tests and thoracolumbar MRI were normal. There were no mutations associated with mitochondrial cytopathy (MELAS nt 3243, MERRFnt8344, NARP nt8993, LHONnt 3460, nt 11778 , nt 14484, nt 15257), and the muscle biopsy findings were normal.

Her neurological examination revealed cachexia, short stature, dysarthria, total ophthalmoplegia, mild ptosis, rigidity in the upper extremities, and flexion contractures and spasticity in the lower extremities. She had muscle weakness $(3 / 5)$, increased deep tendon reflexes in her lower extremities, and bilateral Babinski sign. The patient had normal complete blood count, erythrocyte sedimentation rate, serum 
electrolytes, liver and kidney function tests, vitamin E, 25-OH vitamin $\mathrm{D}$, vitamin B12, folate, thyroid function tests, PTH, ceruloplasmin, 24-hour urine $\mathrm{Cu}$ levels, IgG, IgA, and IgM. The immunological tests for vasculitis (ANA and anti-ds DNA), and autoimmune hepatitis markers (AMA, anti-LKM-1, ASMA) were negative. The patient had normal lactic acid and pyruvic acid levels. New brain MRI showed the

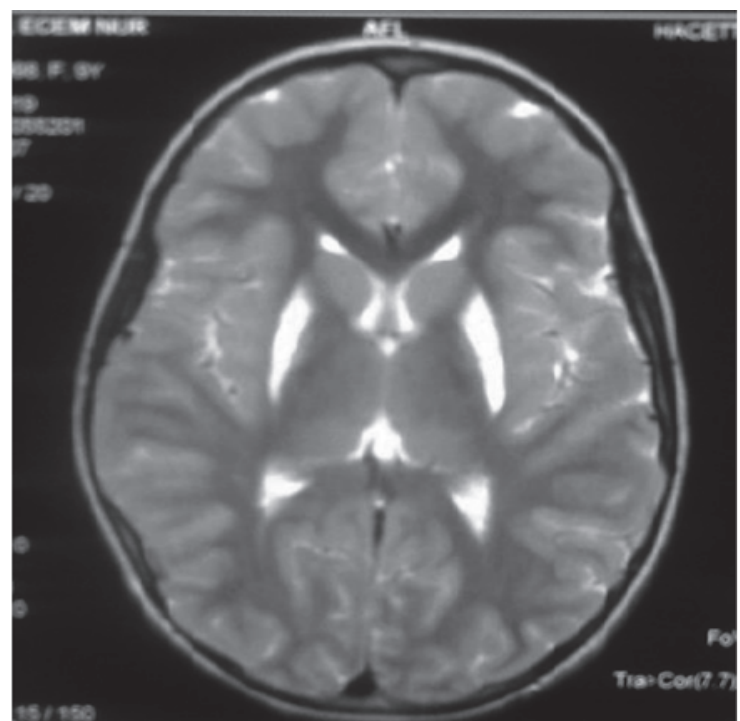

Fig. 1. Axial T2-weighted MRI showing hyperintense and swollen heads of bilateral putamen and caudate nuclei.

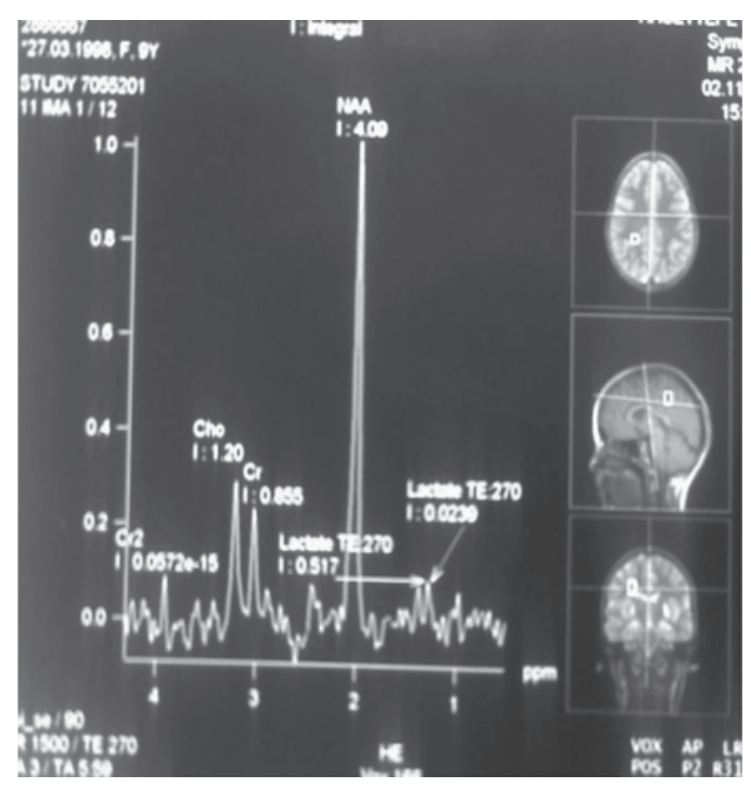

Fig. 2. MRI spectroscopy showing lactate peaks in supraventricular white matter. same findings with the previous one. ENMG performed due to muscle weakness of the lower extremities demonstrated demyelinating neuropathy findings. After exclusion of a number of causes such as Wilson, vasculitis, autoimmune and mitochondrial cytopathy, it was supposed that the findings of the patient (short stature, cachexia, nausea, vomiting, ophthalmoplegia, leukoencephalopathy, peripheral neuropathy) might be compatible with MNGIE. Brainstem auditory evoked response (BAER) was obtained with suspicion of MNGIE, and sensorineural hearing loss was seen. However, since the activity of thymidine phosphorylase was normal, MNGIE was no longer considered. During this period, severe abdominal pain, vomiting, nausea, and weight loss of the patient increased, and we considered presence of a systemic disease. Furthermore, electrolytes and abdominal ultrasound were normal and there was no surgical pathology in this patients with abdominal sensitivity and slight intestine noise (gastrointestinal dysmotility or pseudoobstruction). Endoscopy and biopsy were performed. The histopathological findings were compatible with gluten sensitive enteropathy type III villous atrophy. The levels of the serological markers (AGA IgA and lgG, t-TG IgA) were significantly high. The patient was diagnosed with $\mathrm{CD}$, and a gluten-free diet was administered. Although the patient's external ophtalmoplegia subsided and she gained weight one year after starting the gluten-free diet, MRI showed new lesions in parasagittal white matter (Fig. 3, 4).

\section{Discussion}

Mitochondrial neurogastrointestinal encephalomyopathy (MNGIE) is a rare autosomal recessive mitochondrial disease. It occurs due to a mutation which is responsible for thymidine phosphorylase, and systemic accumulation of thymidine (dThd) and deoxyuridine (dUrd). A number of systems may be involved including central and peripheral nervous systems, and the disease is characterized by short stature, cachexia, and gastrointestinal tract symptoms. The clinical picture is not the same in all patients. The gastrointestinal symptoms (nausea, vomiting, pseudo-obstruction, diarrhea, abdominal pain) are seen in $45-67 \%$ of the patients. It was reported that the symptoms of our patient, 


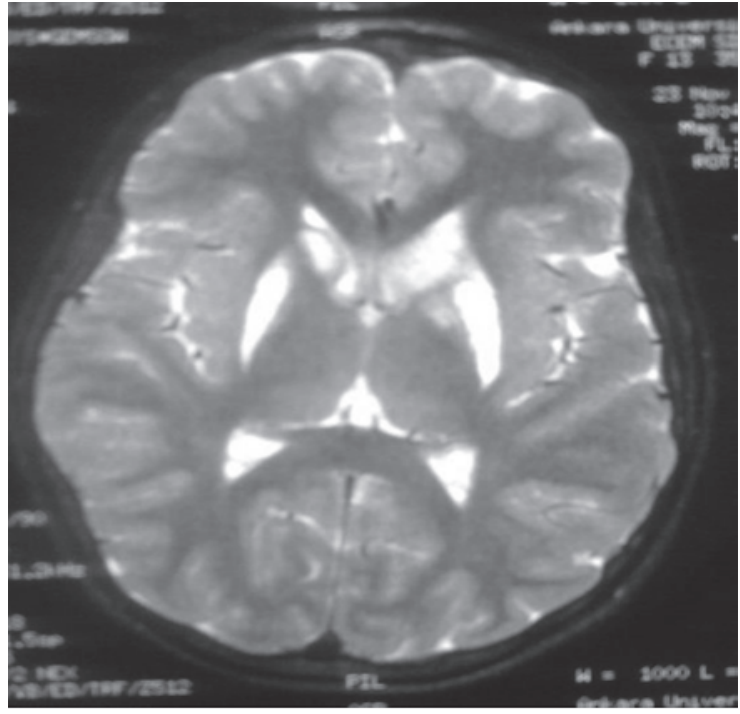

Fig. 3. After gluten-free diet, control brain MRI showed bilateral involvement of putamen on axial T2-weighted images, similar to previous MRI. However, there was increased intensity in bilateral, and predominantly left caudate nucleus.

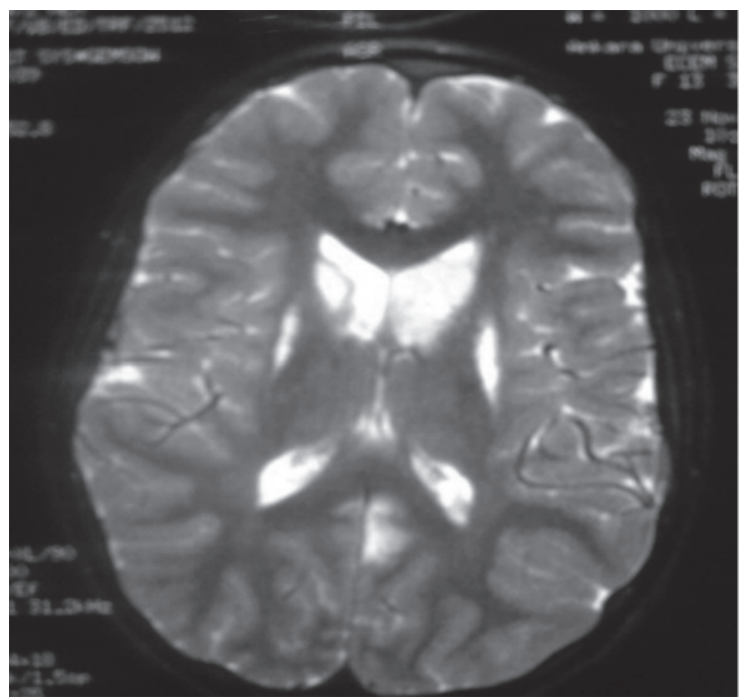

Fig. 4. After gluten-free diet, control brain MRI, axial T2 weighted images showed a new hyperintens lesion improvement in white matter in right parasagital field.

namely ophthalmoplegia, leukoencephalopathy, peripheral neuropathy, and hearing loss, constituted the clinical picture on admission in patients with MNGIE 6 .7. However, the thymidine phosphorylase level was normal in our patient, and we searched for other diseases presenting with a similar clinical picture, and diagnosed our patient as CD. This shows that CD includes all signs of MNGIE, and has a wide range of symptoms. Other important point is the presence of some neurological symptoms in our patient, which were reported to appear in years in cases with CD. Therefore, our patient is a rare and important case for the presence of neurological burden. This aspect of "MNGIE" findings was not reported before.

Celiac disease is a chronic, immune-mediated disorder that primarily affects the gastrointestinal tract. A number of patients, especially adults, may be asymptomatic or have only extraintestinal symptoms at the onset, without any of the classical Celiac symptoms ${ }^{5,8}$.

The relation between $\mathrm{CD}$ and neurological involvement is still unclear 2,3,4. The only histopathological study performed in patients with $C D$ and ataxia showed lymphocytic infiltration in the cerebellum, damage to the posterior columns of the spinal cord, and sparse infiltration of the peripheral nerves ${ }^{9}$. Also, sera from patients with $C D$ and neurological manifestations evoke a mitochondrial-dependent apoptosis in vitro, suggesting that neurotoxic antibodies might be present ${ }^{10}$. Those findings support the hypothesis that neurological manifestations of CD are immune-mediated. The underlying factors of nervous system symptoms are less likely to be associated with celiac disease than a direct complication ${ }^{3-5}$.

Neurological involvement is seen in $6-10 \%$ of $\mathrm{CD}$ patients, and it was first published in $1966^{11}$. Luostarinen et $\mathrm{l}^{12}$. stated that $7 \%$ of the patients presented with neurological findings. The best described neurological complications of $C D$ are ataxia, peripheral neuropathy and epilepsy, and more controversial ones include myopathy, autonomic neuropathy, migraine, cognitive impairment, multiple system atrophy, dystonia, dysarthria, ophthalmoplegia, optic neuritis, multiple sclerosis, difficulty of walking, tremor, white matter and basal ganglion lesions, pyramidal and extra-pyramidal symptoms, childhood stroke, and sensorineural hearing loss $3,4,13-18$.

The MRI findings of our patient progressed despite administration of gluten-free diet, but her neurological findings subsided to some extent. It was reported in the literature that gluten-free diet ameliorated the gastrointestinal symptoms, but it had no effect on neurological findings ${ }^{13,14}$. It appears that further studies are needed to prevent neurological complications of $\mathrm{CD}$ since gluten-free diet is not effective 
on its neurological complications.

In conclusion, after a thorough investigation, we found out that our patient's neurological findings were due to $\mathrm{CD}$, based on duodenal biopsy and positive serum antibodies. Other causes were excluded with appropriate tests. Celiac disease should be kept in mind in patients with undiagnosed neurological findings.

\section{REFERENCES}

1. Tovoli F, Masi C, Guidetti E, Negrini G, Paterini P, Bolondi L. Clinical and diagnostic aspects of gluten related disorders. World J Clin Cases 2015; 3: 275-284.

2. Husby S, Koletzko S, Korponay-Szabo IR, et al. European Society for Pediatric Gastroenterology, Hepatology, and Nutrition Guidelines for the Diagnosis of Coeliac Disease. JPGN 2012; 54: 136-160.

3. Grossman G. Neurological complications of coeliac disease: what is the evidence? Review. Pract Neurol 2008; 8: 77-89.

4. Bürk K, Farecki ML, Lamprecht G, et al. Neurological Symptoms in Patients with Biopsy Proven Celiac Disease. Mov Disord 2009; 24: 2358-2362.

5. Hadjivassiliou M, Sanders DS, Grünewald RA, Woodroofe N, Boscolo S, Aeschlimann D. Gluten sensitivity: from gut to brain. Lancet Neurol 2010; 9: 318-330.

6. Teitelbaum JM, Berde CB, Nurko S, Buonomo C, PerezAtayde AR, Fox VL. Diagnosis and management of MNGIE syndrome in children: case report and review of the literature. J Pediatr Gastroenterol Nutr 2002; 35: $377-383$

7. Garone C, Tadesse S, Hirano M. Clinical and genetic spectrum of mitochondrial neurogastrointestinal encephalomyopathy. Brain 2011;11: 3326-3332.
8. Briani C, Zara G, Toffanin E, et al. Neurological complications of celiac disease and autoimmune mechanisms: preliminary data of a prospective study in adult patients. Ann NY Acad Sci 2005; 1051: 148-155.

9. Hadjivassiliou M, Boscolo S, Davies-Jones GA, et al. The humoral response in the pathogenesis of gluten ataxia. Neurology 2002; 58: 1221-1226.

10. Cervio E, Volta U, Verri M, et al. Sera from patients with celiac disease and neurologic disorders evoke a mitochondrial-dependent apoptosis in vitro. Gastroenterol 2007; 133: 195-206.

11. Cooke WT, Smith WT. Neurological disorders associated with adult celiac disease. Brain 1966; 89: 683-722.

12. Luostarinen L, Pirttilä T, Collin P. Coeliac disease presenting with neurological disorders. Eur Neurol 1999; 42: 132-135.

13. Kieslich M, Errázuriz G, Posselt HG, et al. Brain whitematter lesions in celiac disease: a prospective study of 75 diet-treated patients. Pediatrics 2001; 108: E21.

14. Beyenburg S, Scheid B, Deckert-Schlüter M, Lagrèze HL. Chronic progressive leukoencephalopathy in adult celiac disease. Neurology 1998; 50: 820-822.

15. Yıldırım SV, Tiker F, Barutcu O, Cengiz N. Bilateral calcifications in the basal ganglia, and frontal and parietal lobes of a patient with coeliac disease. Pediatr Radiol 2005; 35: 710-712.

16. Parisi P, Principessa L, Ferretti A, et al. "EEG abnormalities" may represent a confounding factor in celiac disease: A 4-year follow-up family report. Epilepsy Behav Case Rep 2014; 2: 40-42.

17. Gobbi G, Bouquet F, Greco L, et al. Coeliac disease, epilepsy, and cerebral calcifications. Lancet 1992; 340: 439-443.

18. Leggio L, Cadoni G, D’Angelo C, et al. Coeliac disease and hearing loss: Preliminary data on a new possible association. Scand J Gastroenterol 2007; 42: 1209-1213. 